\title{
THE POST ANNEALED EFFECT ON THE OPTICAL PROPERTIES OF $\mathrm{Cu}_{2} \mathrm{ZnSnS}_{4}$ NANOMATERIALS
}

\author{
Bincy John', G. Genifer Silvena², A. Leo Rajesh3, \\ 1,2,3Department of Physics, St. Joseph's College (Autonomous), Tiruchirappalli 620002, Tamilnadu, India . \\ E-mail address: aleorajesh@gmail.com
}

\begin{abstract}
Cost effective photovoltaic $\mathrm{Cu}_{2} Z n \mathrm{ZnS}_{4}$ (CZTS) nanomaterials with kesterite crystal phase were synthesized using a facile solvothermal method. Here ethylene glycol was the solvent and Polyvinylpyrrolidone (PVP) used as capping agent. The obtained CZTS nanoparticles were characterized by means of suitable analytical techniques such as X-ray powder diffraction (XRD), Field Emission Scanning Electron Microscope (FESEM), Raman Spectroscopy, Ultraviolet- Visible (UV-Vis) Spectrometer and Photoluminescence $(\mathrm{PL})$ respectively. The XRD results showed that the obtained sample presented as kesterite crystallinity with (112), (200), (220) and (312) planes of the CZTS nanoparticles and the average crystallite size was about $5.7 \mathrm{~nm}$. The morphological investigation results given by the FESEM revealed that the average particle size of the sample was around 129 to160 $\mathrm{nm}$. In addition, the optical observation suggested by both the absorption of UV-Vis and emission spectra of PL showed that the CZTS nanoparticles produced by this solvothermal method has the direct band gap energy within the range of 1.49 to $1.53 \mathrm{eV}$, which is near the optimal value and it is a dynamic absorber material for the efficient photovoltaic application.
\end{abstract}

Keywords: $\mathrm{Cu}_{2} \mathrm{ZnSnS}{ }_{4}$, Solvothermal, Nanomaterials, Semiconductors, Solar energy materials.

\section{INTRODUCTION}

In search of low cost photovoltaic materials, group $\mathrm{I}_{2}-\mathrm{II}-\mathrm{IV}-\mathrm{V} \mathrm{I}_{4}$ quaternary Chalcogenides offers enormous opportunities as they are abundant in nature, environmentally safe and cost effective. Among the many important functions pursued, tailoring the optical band gap suitable for photovoltaic applications has gained considerable attention [1]. $\mathrm{Cu}_{2} \mathrm{ZnSnS}_{4}$ with an optimal direct band gap of $1.5 \mathrm{eV}$ and a large absorption coefficient over $10^{4} \mathrm{~cm}^{-1}[2,3]$, is one of the most promising material for the absorber layers of thin film solar cells. However the preparation routes of CZTS thin films have been reported, such as sulfurization of sputtered [4] or evaporated [5] stacked films, the spray method [6], the sol-gel method [7] and electrode deposition $[8,9]$, which leads to the current highest performing CZTS solar cells (>11\% efficiency). In all these methods the relative complex devices, such as vacuum system [4] or an electro deposition apparatus are required $[8,9]$.

In this work we report a solution- based simple, low cost, large scale and high throughput solvothermal process. It is a non - vacuum based method and acquires nanostructures with controllable dimensions and aligned morphologies that can potentially provide more efficient charge generation, collection and transfer due to the large junction area and short collection distance to the interface [10]. In this process, the reaction between the process and the precursors and solvent is carried out in a closed system at a temperature higher than that of the boiling point of the temperature. . The reaction temperature had given a great impact on the structure and morphology of the nanoparticles. Consequently, autogenous pressure parameter that involved in the process plays a major role to enhance the chemical reactivity [11]. The obtained CZTS nanoparticles could be used in the form of nanoparticles ink that were coated on a substrate and sintered into bulk material to prepare large -area uniform film with non toxic and low cost materials which acts as a promising p-type candidate for photovoltaic applications.

\section{EXPERIMENTAL PROCEDURE}

Materials and Synthesis

Copper (II) chloride dehydrate $\left(\mathrm{CuCl}_{2}\right.$. $2 \mathrm{H}_{2} \mathrm{O}$ ),zinc(II)chloride $\left(\mathrm{ZnCl}_{2}\right)$ and tin (IV)chloride pentahydrate $\left(\mathrm{SnCl}_{4}, 5 \mathrm{H}_{2} \mathrm{O}\right)$ were all of analytical grade 
and used as received. Thiourea $\left(\mathrm{CH}_{4} \mathrm{~N}_{2} \mathrm{~S}\right)$ was used as a source of sulfur, and Polyvinylpyrrolidone (PVP) was used as structure-directing ligands.

In a typical reaction, for the preparation of CZTS nanopowder, the precursor solution had the composition of atomic ratio $\mathrm{Cu} / \mathrm{Zn} / \mathrm{Sn} / \mathrm{S}$ of $2: 1: 1: 5$. For the reaction, 5 $\mathrm{mm}$ copper (II) chloride dehydrate, $2.5 \mathrm{~mm}$ zinc (II) chloride, $2.5 \mathrm{~mm}$ tin (IV) chloride pentahydrate, $12.5 \mathrm{~mm}$ thiourea was dissolved in ethylene glycol under magnetic stirring for $30 \mathrm{~min}$. Later $2 \mathrm{~g}$ of PVP were added to the above mixture inorder to stabilize the growth and allow the formation of well defined structure without agglomeration. Then the mixed solution was transferred into a Teflon lined stainless steel autoclave with $80 \%$ of its capacity and maintained at $230^{\circ} \mathrm{C}$ for $24 \mathrm{~h}$. After solvothermal reaction, the autoclave was cooled down to room temperature under ambient air. The black precipitate was collected, washed many times with deionized water and ethanol by centrifugation at 4000 rpm for $15 \mathrm{~min}$. The final product was dried over night at $90^{\circ} \mathrm{C}$.

\section{Characterization}

The crystallinity and phase purity of the synthesized CZTS nanoparticles were investigated using $X$-ray Diffraction (XRD, Rigaku) equipped with Cu-K $\beta(\lambda$ $\beta$ radiation $(\lambda=1.39220 \AA)$. The particle size and morphology of the synthesized product was analyzed using field emission scanning electron microscope (FESEM, ZEISS at accelerating voltage $5 \mathrm{KV}$ ). The optical absorption spectra were recorded on a Perkin -Elmer (Model Lambda 35) spectrophotometer. The room temperature Photoluminescence was recorded using Perkin -Elmer (Model Lambda 45) and the Raman analysis was performed using Bruker: RFS 27 respectively.

\section{RESULTS AND DISCUSSION}

The crystal phase of the CZTS nano particles are identified by $X$ - ray diffraction shown in fig.1. Here the scanning range is from $10^{\circ}$ to $90^{\circ}$. The obtained pattern exhibit three broad peaks at $2 \theta=28.44^{\circ}, 47.32^{\circ}$ and $55.82^{\circ}$, that are attributed to the planes (112), (220), (312) respectively. These XRD pattern can be perfectly indexed as CZTS of kesterite structure and has a unit cell as tetragonal (JCPDS, card no. 26-0575) with the cell parameters $a=b=5.434 \AA$ and $c=11.014 \AA$.

According to the Debye -Scherrer formula, the average size of the nanoparticles was determined by

$$
D=\frac{K \lambda}{\beta \cos \theta}
$$

where $\mathrm{K}$ is the Scherrer constant $(\mathrm{K}=0.9), \lambda$ denotes the wavelength of used radiation $\mathrm{Cu}-\mathrm{K} \quad \beta \quad(\lambda$ $\beta$ radiation $(\lambda=1.39220 \AA), \quad \beta$ is the full width at half maximum of the (112) diffraction peaks and $\theta$ is the Bragg angle. The average crystallite size of CZTS is calculated to be $5.7 \mathrm{~nm}$. As the diffraction peaks that are observed in the pattern matches well simultaneously with that of $\mathrm{ZnS}$ (65-1691) and $\mathrm{Cu}_{2} \mathrm{SnS}_{3}$ (27-0198), making it difficult to distinguish. Hence Raman spectroscopy techniques are used to identify the secondary phase.

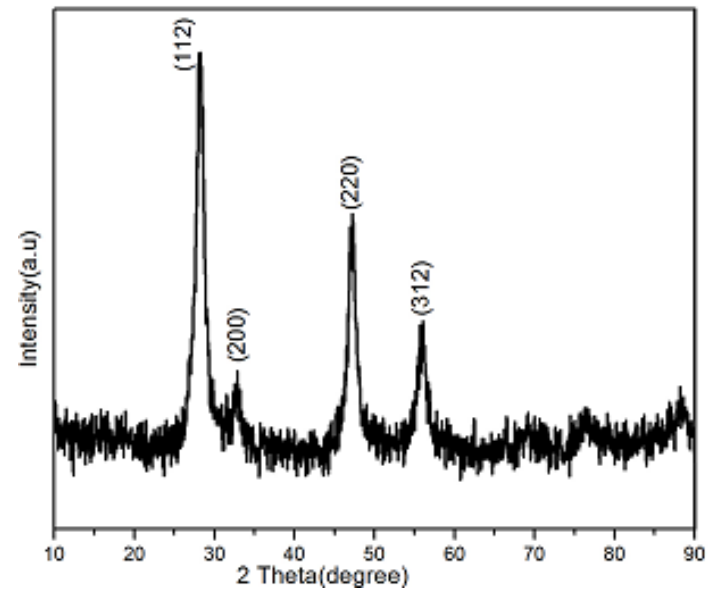

Fig. 1. XRD pattern of the obtained CZTS nanoparticles.

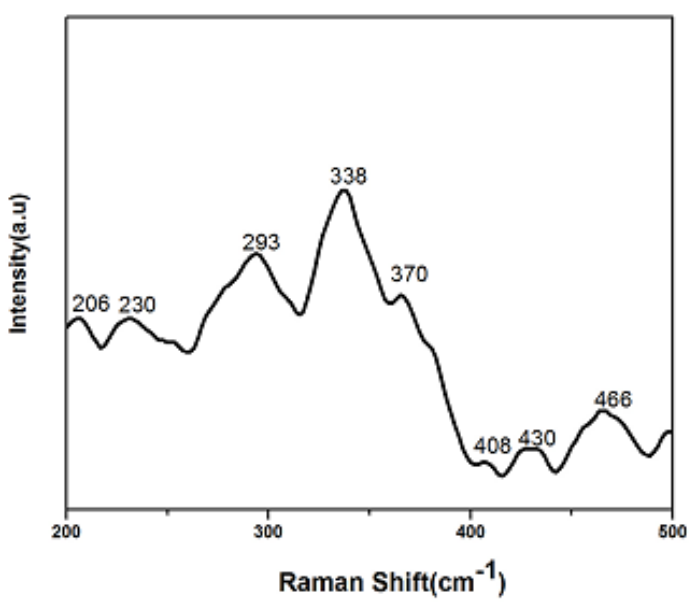

Fig.2. Raman Spectra of the as -synthesized CZTS nano particles 
Fig. 2. give the Raman spectra of the CZTS nanoparticles. The existence of CZTS are confirmed by the Raman shift at $338 \mathrm{~cm}^{-1}$ and $293 \mathrm{~cm}^{-1}$ are in consistence of the CZTS Raman data reported by previous study[12], and most importantly the absence of shifts at $275 \mathrm{~cm}^{-1}, 352 \mathrm{~cm}^{-1}$ and $267 \mathrm{~cm}^{-1}, 303 \mathrm{~cm}^{-1}, 365$ $\mathrm{cm}^{-1}$ suggests the absence of cubic $\mathrm{ZnS}$ and $\mathrm{Cu}_{2} \mathrm{SnS}_{3}$, respectively. Therefore, the XRD data must correspond to CZTS [13].

The surface morphology of the synthesized CZTS nanoparticles is obtained from the FE-SEM images with low and high magnifications are shown in fig.3. From figure we can see that the CZTS nanoparticles are composed of a large number of uniform spheres like particles with average size of $129 \mathrm{~nm}$ to $160 \mathrm{~nm}$.
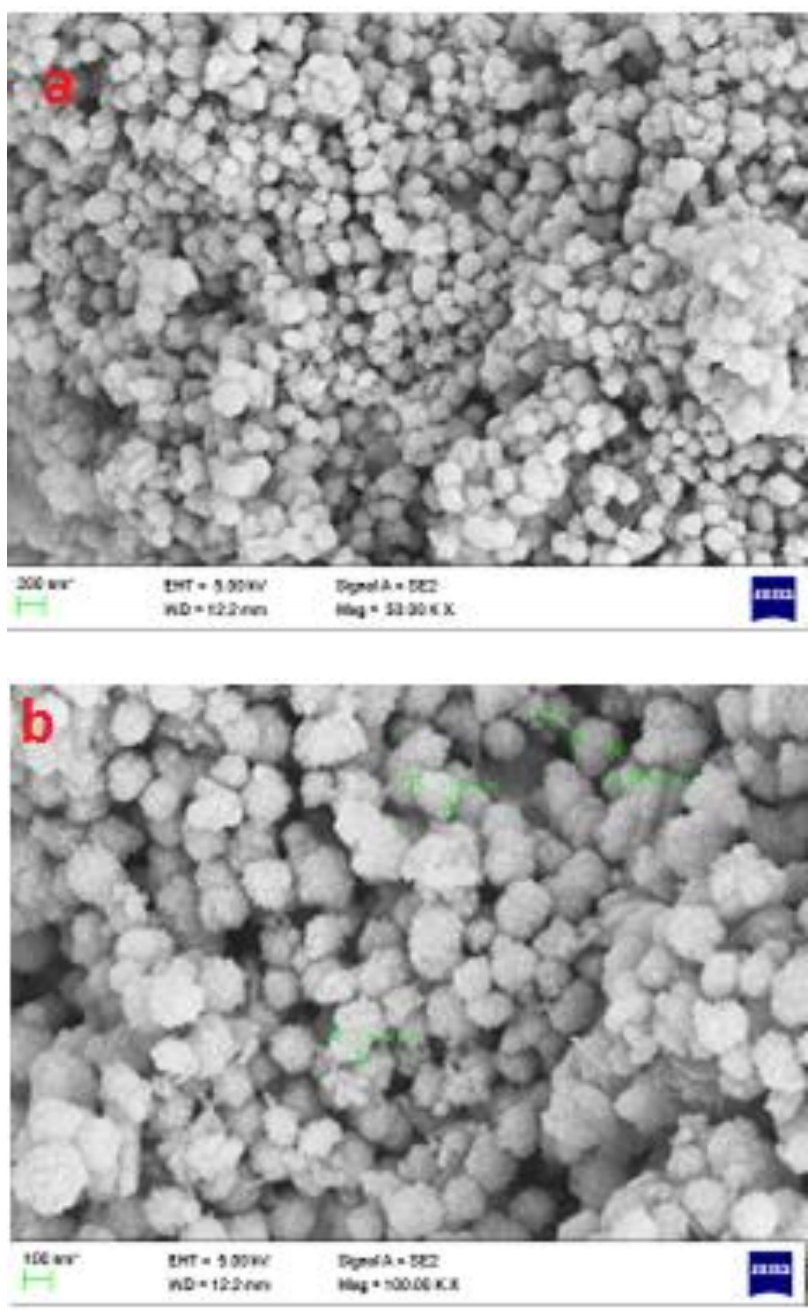

Fig. 3. FESEM images of the obtained CZTS nanoparticles with (a) low and (b) high magnifications.

The optical properties of the typical CZTS nano products are characterized by UV-Vis spectroscopy and
Photoluminescence. Before doing anneling and post annealed CZTS nanoparticles at $110^{\circ} \mathrm{C}$ for $2 \mathrm{hrs}$ are taken for the optical analysis. Before doing the characterization the nano powder is completely dispersed in ethylene glycol by sonication. Fig .4.(a) shows the absorbance spectrum of the CZTS nanoparticles before anneling. Here we can see that the pre- annealed nano powder has obtained higher absorption in UV and near IR region and that weak absorption in the visible region. The band gap energy can be determined by extrapolating straight line of the plot $(\alpha h v)^{2}$ versus $(h v)$ as shown in the inset of fig.4.(a) ( $\alpha=$ absorbance, $\mathrm{h}=$ Plank's constant, and $v=$ frequency). The band gap energy of the present sample is $1.38 \mathrm{eV}$. Fig. 4(b) gives the absorbance spectrum of the post annealed CZTS nanoparticles.
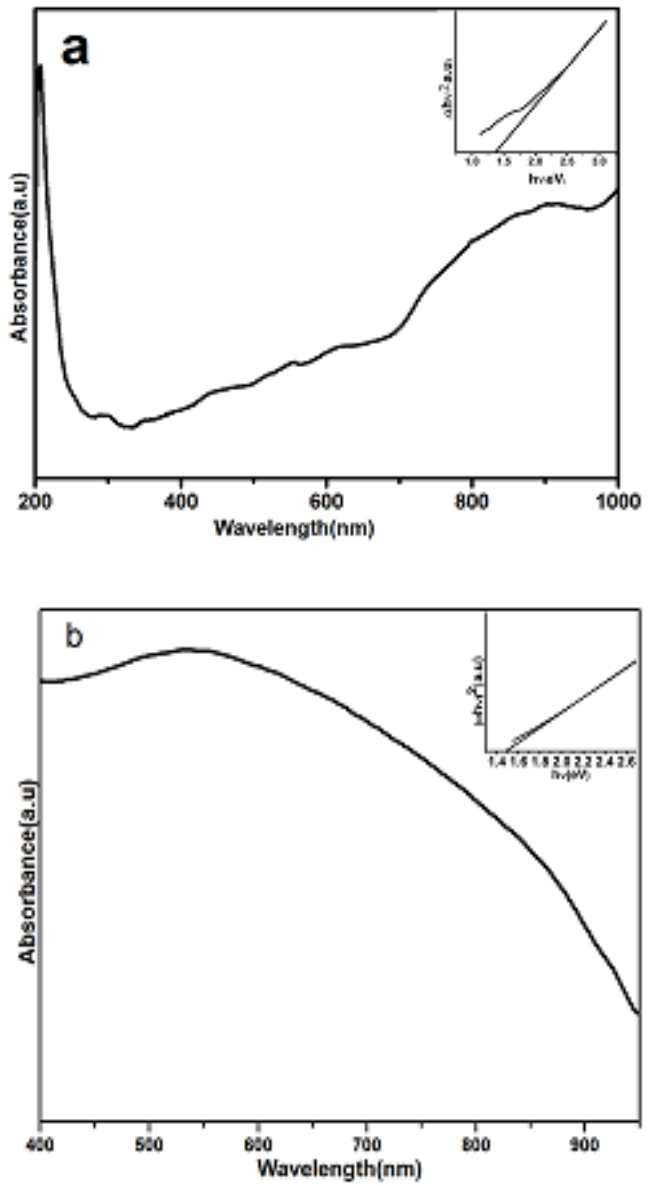

Fig.4. UV-Vis absorbance spectra of CZTS nano particles (a) before annealing (The inset image shows obtained band gap energy of 1.38 eV). (b)Post annealing (The inset image shows obtained band gap energy of $1.49 \mathrm{eV})$.

In this case we can clearly see that when temperature increases the conductivity increases and thus obtaining a 
broad absorption spectrum in the visible region. The direct optical band gap energy for this sample is $1.49 \mathrm{eV}$ which is shown in the inset of the fig. 4(b). In both cases the optical absorption coefficient of the CZTS nano particles are around $10^{6} \mathrm{~cm}^{-1}$.

Fig. 5 shows the PL spectra of CZTS nano particles. Post annealed CZTS nano particles exhibit emission in the range of $1.47 \mathrm{eV}$ to $1.57 \mathrm{eV}$ and the spectra is dominated by an intense exciton- related emission at 1.53 $\mathrm{eV}$ which is shown in fig. 5(a). Whereas the spectra that shown in fig 5. (b) has determined without heating obtains emission in the range of $1.504 \mathrm{eV}$ to $1.56 \mathrm{eV}$ with an intense exciton emission at $1.52 \mathrm{eV}$. The post annealing improves the optical properties of the CZTS nanoparticles by increasing the band gap energies of UVVis to $1.49 \mathrm{eV}$ and $\mathrm{PL}$ emission to $1.53 \mathrm{eV}$ which are consistent with the literature value of 1.45-1.6 eV [14-16].
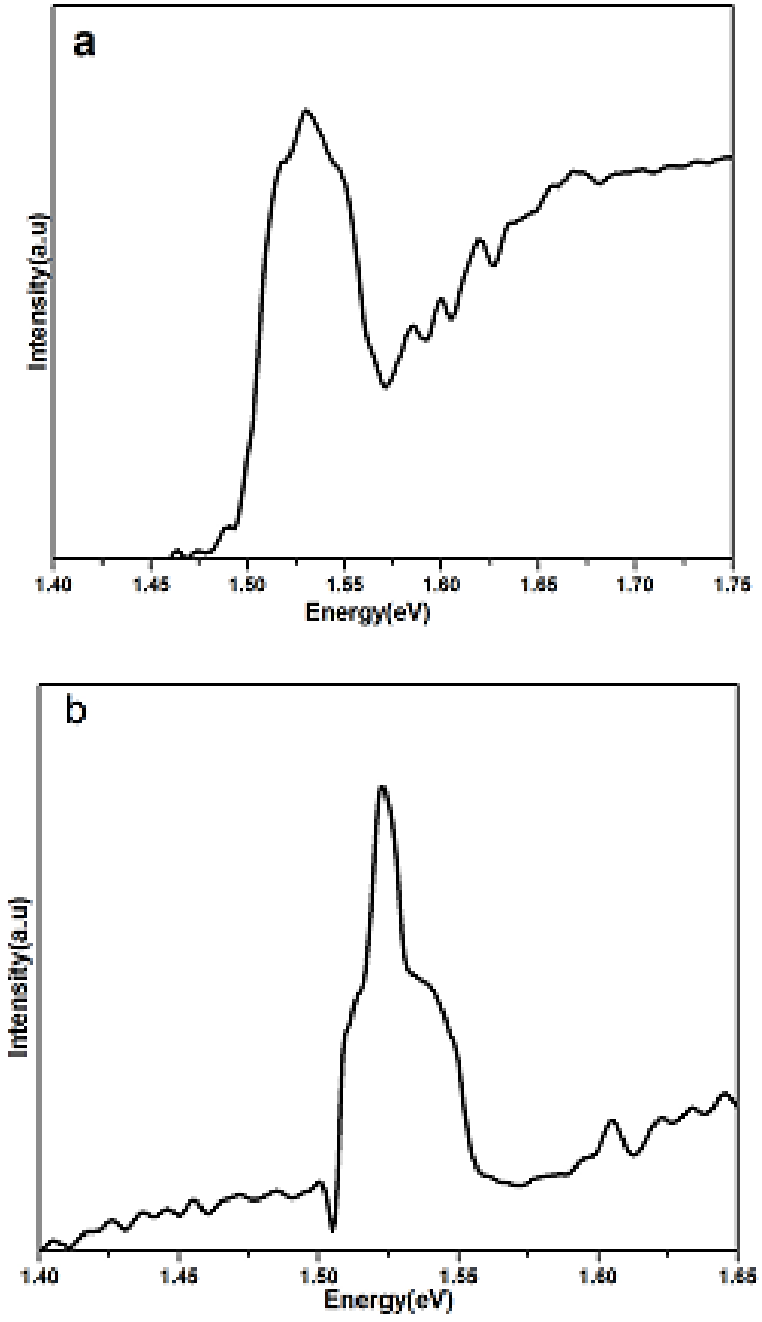

Fig.5. Photoluminescence of CZTS nano particles (a) Post annealing (b) Before annealing.

\section{CONCLUSIONS}

In conclusion, CZTS nanoparticles with kesterite structure were successfully synthesized via a facile solvothermal method. The structure, composition and morphology of the as- synthesized nano powder was confirmed by XRD, Raman scattering and FE-SEM characterizations. The post annealed effect on the optical properties of the CZTS nano materials improves the band gap energies in both UV-Vis absorption spectra and PL emission spectra. The direct band gap energy of the UVVis absorption was $1.49 \mathrm{eV}$ and that of PL emission was $1.53 \mathrm{eV}$ which were optimal for photovoltaic applications.

\section{ACKNOWLEDGEMENT}

The authors would like to thank CENSe, IISc Bangalore for carrying out the XRD and FE-SEM measurements respectively.

\section{REFERENCES}

[1] Meenakshi Gusain, Pooja Rawat, R. Nagarajan, "Solvent mediated rapid synthesis of orthorhombic $\mathrm{Cu}_{2} \mathrm{ZnSnS} 4$ (CZTS)", Mater. Lett., vol.133, 2014, pp. 220-223.

[2] K.Ito, T. Nakazawa, "Electrical and optical properties of stannite-type quaternary semiconductor thin films", Jpn. J. Appl. Phys., vol. 27, no. 11, 1988, pp. 2094-2097.

[3] H. Katagiri, K. Saitoh, T. Washio, H. Shinohara, T. Kurumadani, and S. Miyajima, "Development of thin film solar cell bas/ed on $\mathrm{Cu}_{2} \mathrm{ZnSnS}_{4}$ thin films," Sol. Energy Mater. Sol. Cells, vol. 65, no. 1, 2001, pp. 141-148.

[4] T. Tanaka, T. Nagatomo, D. Kawasaki, M. Nishio, Q.X Guo, A. Wakahara, et al., "Preparation of Cu2ZnSnS4 thin films by hybrid sputtering" J. Phys. Chem. Solids ,vol. 66, 2005, pp. 1978.

[5] Weber, H. Krauth, S. Perlt, B. Schubert, I. Kotschau, S. Schorr, et al , "Multi-stage evaporation of $\mathrm{Cu}_{2} \mathrm{ZnSnS}_{4}$ thin films", Thin Solid Films, vol. 517, iss. 7, 2009, pp. 25242526.

[6] N. Kamoun, H .Bouzouita, B. Rezig, "Thin film solar cells from earth abundant materials: Growth and characterization of $\mathrm{Cu}{ }_{2} \mathrm{ZnSn}(\mathrm{S} \mathrm{Se})_{4}$ thin films and their solar cells", Thin Solid Films, vol .515, 2007, pp. 5949.

[7] Kunihiko Tanaka, Masatoshi Oonuki, Norkio Moritake, Hiso Uchiki, "Cu2ZnSnS4 thin film solar cells prepared by nonvacuum processing", Sol. Energy Mater. Sol. Cells, vol. 93, iss.3, 2009, pp. 583.

[8] Ennaoui, M. Lux-Steiner, A Weber, D. Abou-Ras, I. Kotschau, H.W Schock, et al., "Thin film solar cells from earth abundant materials: Growth and characterization of $\mathrm{Cu}_{2} \mathrm{ZnSn}(\mathrm{S} \mathrm{Se})_{4}$ thin films and their solar cells", Thin Solid Films, vol . 517, 2009, pp. 2511. 
[9] J. Minsung, S. Tomohiro, S. Shoso, "Cu2ZnSnS 4 thin films and nanowires prepared by different single-step electrodeposition method in quaternary electrolyte", Mater. Lett., vol.65, issues. 15-16, 2011, pp. 2364-2367.

[10] Liu X, Wang C, Xu J, Zou R, Ouyang L, Xu X, Chen X and Xing $H$, "Fabrication of $\mathrm{ZnO} / \mathrm{CdS} / \mathrm{Cu}_{2} \mathrm{ZnSnS} \mathrm{S}_{4} \mathrm{p}-n$ heterostructure nanorod arrays via a solution-based route", Crystengcomm, vol.15, 2013, iss.6, pp. 1139-45.

[11] G. Demazeau, "Solvothermal processes: new trends in materials chemistry", J. Phys. Conf. Ser., vol.121, iss.8, 2008.

[12] Fernandes PA, Salome PMP, da Cunha AF, "Study of polycrystalline Cu 2ZnSnS 4 films by Raman scattering", J Alloys Compd, vol. 509, iss. 28, 2011, pp. 7600-7606.

[13] Ming Wei,Qingyang Du, Dacheng Wang, Weifeng Liu, Guoshun Jiang, Changfei Zhu, "Synthesis of spindle-like kesterite Cu2ZnSnS4 nanoparticles using thiorea as sulfur source", Mater. Lett. , vol. 79, 2012, pp. 177-179.

[14] S. Thiruvenkadam, D. Jovina, A. Leo Rajesh, "The influence of deposition temperature in the photovoltaic properties of spray deposited CZTS thin films", Sol. Energy, vol. 106, 2014, pp. 166-170.

[15] H. Katagiri, N. Sasaguchi, S. Hando, S. Hoshino, J. Ohashi, and T. Yokota, "Preparation and evaluation of $\mathrm{Cu}_{2} \mathrm{ZnSnS} 4$ thin films by sulfurization of e-beam evaporated precursors," Sol. Energy Mater. Sol. Cells, vol. 49, no. 1-4, 1997, pp. 407-414.

[16] Park J, Song M, Jung WM, Lee WY, Kim H, Kim Y, et al. "Syntheses of Cu2SnS3 and Cu2ZnSnS4 nanoparticles with tunable $\mathrm{Zn} / \mathrm{Sn}$ ratios under multi bubble sonoluminescence conditions", Dalton Transactions, vol.42,iss.29, 2013, pp.10545-50. 